\title{
Nursing care of the patient undergoing lumbar spinal fusion
}

\author{
Maureen P. Lall* \\ Neuroscience and Spine Center, Houston Methodist Sugar Land Hospital, Sugar Land, TX, United States
}

Received: June 22, 2017

DOI: $10.5430 /$ jnep.v8n5p44
Accepted: July 30, 2017

URL: https://doi.org/10.5430/jnep.v8n5p44

\begin{abstract}
Lumbar spinal fusion is a surgical procedure performed to join — or fuse- 2 or more vertebrae in the low back. The procedure is done to stabilize the spine and prevent damage to the cauda equina and emanating nerve roots. Lumbar fusion is commonly indicated for patients with vertebral fractures, infection, or spinal tumors, and it may be appropriate for select patients with degenerative disorders and spinal stenosis. Nurses who care for patients undergoing lumbar fusion require an understanding of lumbar spinal anatomy, spinal pathology, surgical indications, and diagnostic modalities. Knowledge of the distinct surgical approaches and their respective advantages and disadvantages allows nurses to individualize patient care and be alert to postoperative complications. This article reviews clinical and research literature regarding lumbar fusion, with an emphasis on the role of the nurse in promoting a safe perioperative course.
\end{abstract}

Key Words: Lumbar fusion, Spinal surgery, Fusion complication, Spinal pathology, Surgical outcome

\section{INTRODUCTION}

Nurses have an essential role in promoting a safe and efficacious perioperative course for patients undergoing lumbar spinal fusion. This commonly performed surgical procedure prevents movement in the low back by joining-or fusing- 2 or more vertebrae in the lumbar spine. It is indicated for a variety of conditions, ranging from traumatic injuries to degenerative conditions. Since lumbar fusion is reserved for patients for whom conservative, non-operative treatment is not appropriate or has failed, many patients present with complex health care needs. Patients often have chronic pain, physical disabilities, and medical comorbidities that increase the risk of surgical complications. Nevertheless, with expert care from an interdisciplinary team of health care professionals, most patients will experience a decrease in pain and dysfunction following surgery. This article presents an overview of lumbar fusion, including a review of lumbar spinal anatomy, the preoperative evaluation, surgical indications, operative techniques, and postoperative recovery. Specific recommendations to support evidence-based nursing care of patients undergoing lumbar fusion are presented.

\section{LUMBAR SPINAL ANATOMY}

The lumbar spine is comprised of 5 vertebrae, 3 major ligaments, 5 intervertebral discs, and the cauda equina. Each vertebra consists of 2 main elements. The solid anterior or ventral element is called the vertebral body; the posterior or dorsal element is called the arch. The arch is composed of 2 pedicles and 2 laminae that support 7 processes (i.e., 4 articular, 2 transverse, and 1 spinous process). The inferior and superior articulating processes form the facet joints. Collectively, the elements of the arch enclose the spinal foramen,

\footnotetext{
*Correspondence: Maureen P. Lall; Email: mplall@ houstonmethodist.org; Address: Neuroscience and Spine Center, Houston Methodist Sugar Land Hospital, Sugar Land, TX, United States.
} 
maintain spinal alignment, enable movement of the vertebral column, and protect the cauda equina (see Figure 1). Adjacent to the pedicles and facet joints are the neural foramina through which nerve roots exit the cauda equina. ${ }^{[1]}$ Three major ligaments support the spine: (1) the anterior longitudinal ligament, (2) the posterior longitudinal ligament, and (3) the ligamentum flavum. The anterior longitudinal ligament attaches to the anterior surface of the vertebral bodies and intervertebral discs. The posterior longitudinal ligament attaches to the posterior surface of the vertebral bodies and supports the spinal canal. The ligamentum flavum connects the laminae of adjacent vertebrae. Intervertebral discs are avascular structures composed of a central nucleus pulposus and a surrounding annulus fibrosis.

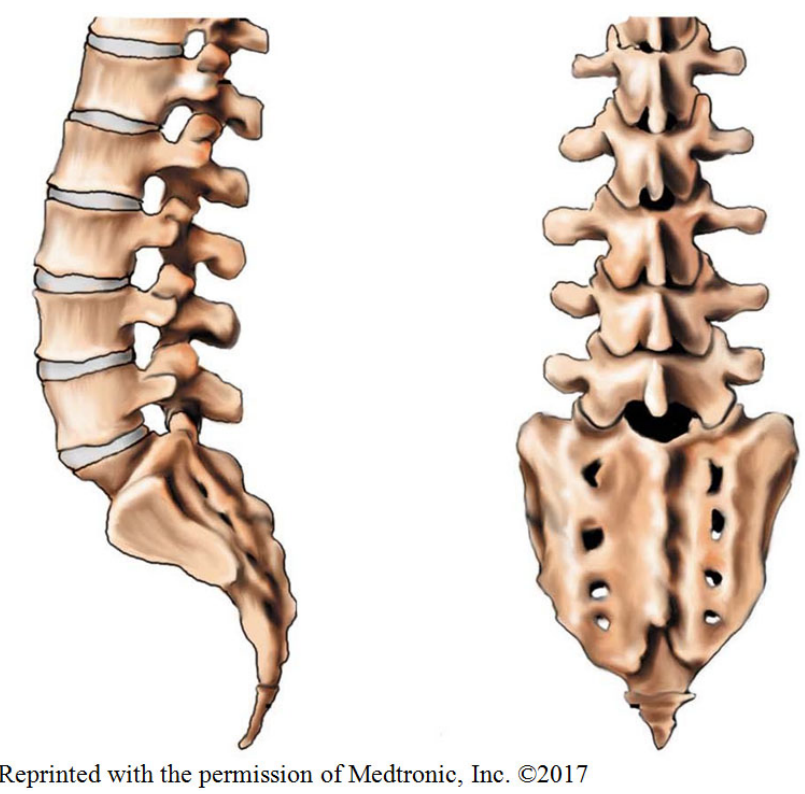

Figure 1. Lumbosacral spine

Intervertebral discs are positioned between vertebral bodies and cushion movement and prevent excessive translation during spinal column movement. ${ }^{[1]}$ A thin, cartilaginous endplate bonds each intervertebral disc to the adjacent vertebral bodies and helps the disc maintain its shape and resist herniation. ${ }^{[1]}$ The spinal cord is a mass of nerve tissue that occupies the vertebral canal. The cord typically ends with the conus medullaris at the level of T12 to L1. A long, fanning bundle of nerve roots, collectively known as the cauda equina, emanates from the end of the spinal cord. The lumbar nerve roots innervate the lower extremities and the sacral nerve roots control bowel, bladder, and sexual function. The spinal cord is covered by 3 meninges: the dura mater, the arachnoid, and the pia mater. The outermost dura mater is susceptible to injury during posterior lumbar fusion, potentially resulting in a dural tear. The innermost subarachnoid space contains cerebrospinal fluid, which cushions the cord and nourishes the neural components. Between the meninges exist potential spaces: the epidural space, the subdural space, and the subarachnoid space. Potential spaces may be involved in fusion complications, such as hematomas and abscesses. ${ }^{[1]}$

\section{Preoperative evaluation}

Neurosurgeons and orthopedic spine surgeons perform lumbar fusion. They learn the procedure during residency training and spine-focused fellowship programs. The 2 specialty groups utilize many of the same surgical techniques and have similar 30-day readmission and mortality rates following spine surgery. ${ }^{[2]}$ However, an important distinction between the specialties is that typically only neurosurgeons operate on patients with intradural pathology, such as spinal cord tumors.

\subsection{History and physical examination}

The preoperative evaluation commences with the history and physical examination. The location and duration of pain, the frequency and characteristics of accompanying symptoms, and the presence and level of neurological impairment often suggest a specific spinal anomaly. Radiculopathy (i.e., pain and/or paresthesias in the distribution of a nerve root), motor weakness, and reflex asymmetry, combined with a positive straight leg raise, suggest lumbar disc herniation. ${ }^{[1,3]}$ Leg pain that exacerbates with lumbar extension (i.e., standing upright or walking downhill) and improves with lumbar flexion (i.e., leaning on a grocery cart or sitting) suggests spinal stenosis. ${ }^{[1,3]}$ However, since many non-spinal anomalies, such as vascular claudication, present with similar symptoms, a thorough evaluation is necessary to rule out other conditions.

The preoperative evaluation also reviews prior treatment interventions, such as physical therapy and spinal injections. Patient response to these interventions often yields diagnostic clues and ensures that appropriate conservative measures have been trialed prior to fusion surgery. Exhausting conservative measures is important because lumbar fusion does not necessarily result in superior outcomes when compared to non-operative treatment. One review of the literature found fair evidence that lumbar fusion was no better than intensive, interdisciplinary rehabilitation programs in reducing pain and improving function in patients with non-radicular lower back pain with degenerative changes. ${ }^{[4]}$ Another literature review cited poor quality evidence in failing to conclude whether surgery resulted in better outcomes compared to non-operative treatment in patients with lumbar spinal stenosis. ${ }^{[5]}$ 


\subsection{Diagnostic imaging and neurophysiologic testing}

Radiological images should be available for the surgeon's review during the initial evaluation. However, if patients do not have recent imaging, it may be acceptable to defer imaging until after the evaluation. This allows the surgeon to order the most appropriate imaging based on suspected pathology and avoids redundant or unnecessary studies.

Plain radiography is used to assess bony structures, spinal alignment, and vertebral fractures. Flexion and extension radiographs are used to assess segmental instability. When more detailed spinal images are needed, computed tomography (CT) may be indicated. Like plain radiography, CT uses ionizing radiation; however, unlike plain radiography, $\mathrm{CT}$ can distinguish the soft tissues of the spine (i.e., discs, nerve roots, and ligaments) by interpreting the attenuated values of ionized radiation as it passes through tissues of variable density ${ }^{[6]}$ When a mass or fluid collection is suspected, intravenous contrast administered prior to $\mathrm{CT}$ examination can highlight suspected abnormalities. When a tumor or trauma is suspected, CT examination combined with myelography can identify spinal cord compression or localize a spinal lesion relative to the dura. ${ }^{[6]}$ Magnetic resonance (MR) imaging also yields detailed spinal images. MR uses a pulsed radiofrequency beam and a strong magnetic field to produce images, thus avoiding ionizing radiation. When a mass, epidural scarring, abnormal tissue, or a fluid collection is suspected, intravenous gadolinium, or another paramagnetic contrast agent, administered prior to MR will yield sharper images. ${ }^{[6]}$ However, patients with ferromagnetic devices (i.e., artificial pacemakers, some implanted stimulators of the brain or spinal cord, prosthetic devices, metal bullets, and old ferromagnetic intracranial aneurysm clips) cannot undergo MR because the strong magnet can cause the device to malfunction or overheat. Historically, patients with obesity and claustrophobia were also considered unsuitable for MR; however, the availability of wide-bore and open MR units has largely eliminated this limitation.

Neurophysiological testing may be indicated as part of the diagnostic evaluation. Nerve conduction studies and electromyography (EMG) are performed using surface electrodes and needle electrodes, respectively, and assess sensory and motor abnormalities, such as lumbosacral radiculopathy. ${ }^{[1]}$

\section{SURGical INDiCATIONS}

A comprehensive evaluation enables the surgeon to determine the need for lumbar fusion. Traumatic injuries, spinal deformity, and infection or tumors causing spinal instability frequently necessitate fusion. Degenerative spinal conditions may also warrant lumbar fusion and are the leading indication for lumbar fusion in the United States. ${ }^{[7-9]}$

\subsection{Degenerative disc disease}

Degenerative disc disease (DDD) results from age-related changes in the lumbar spine. In a healthy disc, the inner nucleus pulposus has a high water content that allows the disc to act as a shock absorber and distribute mechanical stress and compressive forces in the lumbar region. However, as the nucleus pulposus ages, it loses water content and becomes less elastic. Physical stress in the lumbar spine is then disproportionately borne by the outer annulus fibrosis, which can lead to annular tearing. Gas can also accumulate inside a degenerating disc, which can cause annular bulging. As the degenerating disc loses height and becomes compressed, adjacent bony structures come into direct contact with each other and the ligamentum flavum may buckle. ${ }^{[1]}$ These processes cause further degeneration due to endplate fissuring and osteophyte formation. ${ }^{[1]}$ However, even in the presence of extensive degenerative changes, many degenerative discs are asymptomatic and do not require surgical excision. Surgical intervention should only be considered when symptomatic DDD is accompanied by neurological deficits or when symptomatic DDD fails to respond to at least 6 months of non-operative treatment. ${ }^{[1,3,10]}$

\subsection{Spinal stenosis}

Lumbar spinal stenosis refers to a narrowing of the spinal canal. It can affect the central portion of the canal, the lateral recesses underneath the facet joints, or the foramen (see Figure 2). Although stenosis can be a congenital condition or the result of an inflammatory process, it is frequently associated with space-occupying, degenerative lesions, such as hypertrophic ligamentum flavum, osteophytes, and disk herniation and bulges. Spinal stenosis often has an insidious onset with patients describing a gradual decrease in their ability to walk long distances. Patients may also present with hyperreflexia or absent lower extremity reflexes. ${ }^{[1,3]}$ Many patients with spinal stenosis are successfully treated with surgical decompression at the level of stenosis without fusion. However, when a patient with lumbar spinal stenosis becomes symptomatic and the affected vertebral segment shows signs of instability, fusion is indicated to maintain foraminal height and relieve nerve root compression. ${ }^{[3]}$

\subsection{Spondylolisthesis}

Spondylolisthesis is the slipping or sliding of 1 vertebra on another vertebra. There are 2 primary types of spondylolisthesis, each of which presents with distinguishing signs and symptoms. Degenerative spondylolisthesis most commonly affects the L4-L5 level in older adults and isthmic spondylolisthesis most commonly affects the L5-S1 level in middle-aged adults. ${ }^{[3]}$ Compared to other fusion indications, patients undergoing lumbar fusion for spondylolisthesis tend 
to have better outcomes. ${ }^{[3]}$ One study reported that patients undergoing fusion for spondylolisthesis had shorter periods of postoperative opioid pain reliever use and were more likely to return to work than were patients having fusion for DDD. ${ }^{[11]}$

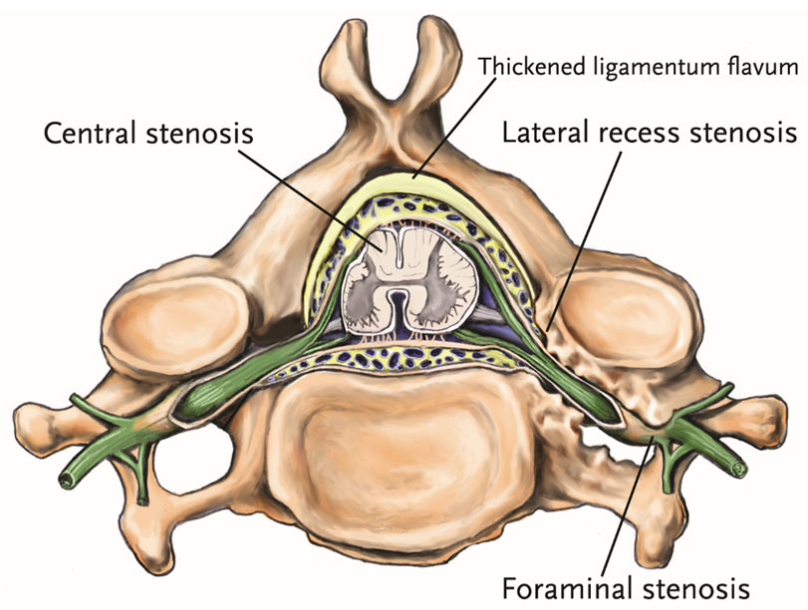

Reprinted with the permission of Medtronic, Inc. C2017

Figure 2. Lumbar spinal stenosis

\section{OPERATIVE APPROACHES}

The surgeon considers the anatomical defect, comorbidities, and the goals of surgery in deciding among several approaches to lumbar fusion. Each approach carries unique risks and benefits and has implications for postoperative management. However, the shared goal of surgery is to stabilize the spine through arthrodesis. The surgeon typically uses graft material combined with graft extender, bone void filler, or a graft supplement to promote new bone growth between 2 or more vertebral segments. Autograft refers to the patient's own bone and is typically harvested from the iliac crest; allograft refers to sterilized bone from a human tissue bank. Both types of graft encourage new bone growth that will lock the unstable vertebral segments together into a solid mass of new bone. Following placement of the graft, the surgeon immobilizes the spinal segment with internal fixation devices (i.e., pedicle screws, rods, or cages containing graft material). Once these elements are secured, osteoblasts should adhere to the exposed bony structures and new bone should grow across the defect. This process occurs over several months and must occur in each fused segment to yield long-term stability. ${ }^{[12]}$

\subsection{Posterior fusion}

There are 2 main types of posterior lumbar fusion: posterolateral fusion and interbody fusion. In posterolateral fusion, the surgeon places the graft between the transverse processes so that new bone grows and fuses the transverse process of

Published by Sciedu Press one vertebra to the transverse process of another vertebra. In interbody fusion, the surgeon removes the intervertebral disc, strips the cartilaginous endplates from the adjacent vertebra, scrapes the endplates to expose bleeding bone, and places the graft between the vertebral bodies so that bone grows and fuses one vertebral body to another vertebral body.

During posterior lumbar interbody fusion (PLIF), the surgeon accesses the spine through a midline incision in the patient's lumbar region. This approach provides the surgeon with direct access to the disc space, which facilitates bilateral decompression and interbody fusion through one incision. ${ }^{[13]}$ Alternatively, the surgeon may perform transforaminal lumbar interbody fusion (TLIF). TLIF carries less risk of neural injury than PLIF because it requires less neural traction. ${ }^{[13]}$ Both posterior approaches avoid the risks of vascular injury, abdominal wall injury, and autonomic complications that are associated with the anterior approach.

\subsection{Anterior lumbar interbody fusion}

During anterior lumbar interbody fusion (ALIF), the surgeon accesses the spine through the abdominal cavity and obtains direct access to the ventral surface of the vertebrae and disc spaces. The anterior approach avoids dissection of the paraspinal musculature and posterior ligaments and is associated with earlier ambulation, less postoperative pain, and shorter inpatient length of stay than the posterior approach. ${ }^{[14,15]}$ However, the anterior approach is also associated with increased risk of vascular complications, visceral injury, retrograde ejaculation, and sympathetic dysfunction because of the proximity of the major vessels, abdominal organs, and hypogastric plexus to the surgical dissection. ${ }^{[13,16]}$ Severe obesity or extensive scarring from previous abdominal surgery may preclude the anterior approach. ${ }^{[13]}$

\subsection{Anterior and posterior approach fusion}

In select cases, the surgeon uses a combined anterior and posterior approach to lumbar fusion (APF). The combined approach provides greater mechanical stability and improves the likelihood of a solid fusion. However, APF is associated with longer operative time, greater intraoperative blood loss, more complications, and longer length of stay. ${ }^{[13,14]}$ Consequently, this approach as a percentage of all lumbar fusions is decreasing in frequency. In 2002, APF comprised $10 \%$ of all lumbar fusions; by 2010, APF made up less than $1 \%$ of all lumbar fusions. ${ }^{[14]}$

\subsection{Extreme lumbar interbody fusion}

In extreme lumbar interbody fusion (XLIF), the surgeon uses a lateral trans-psoas approach to access the surgical site. Similar to ALIF, XLIF facilitates a more extensive discectomy and exposes a larger area for intervertebral graft positioning 
than posterior approaches. However, XLIF is less invasive than ALIF and does not necessitate retraction of the major vessels or sympathetic chain. ${ }^{[15]}$ Retroperitoneal scarring or anomalous vascular anatomy, as well as the need to incorporate L5-S1 into the fusion, may preclude XLIF. ${ }^{[16]}$

\subsection{Minimally invasive surgery}

Minimally invasive surgery (MIS) has emerged as a popular alternative to traditional open lumbar fusion. During MIS, the surgeon performs decompression and fusion while leaving the paraspinal muscles, anterior and posterior longitudinal ligaments, and bony structures largely intact. This is accomplished by using a muscle-splitting approach, such as the Wiltse approach, rather than a direct midline incision. When using the Wiltse approach, the surgeon uses a far lateral entry point and splits the sacrospinalis muscle between the multifidus and longissimus to access the operative site. ${ }^{[17]}$ In comparison to open approaches, MIS is associated with less intraoperative blood loss, shortened length of stay, reduced postoperative opioid use, faster ambulation, accelerated return to work, and long-term improvements in pain, disability, and quality of life. ${ }^{[13,18-20]}$ However, because the surgical field is restricted, MIS is more technically challenging than open surgery. MIS is also associated with greater fluoroscopic exposure and longer operative times. ${ }^{[20]}$

\section{LUMBAR FUSION COMPLICATIONS}

Diligent postoperative care is essential following lumbar fusion because up to $30 \%$ of patients experience an adverse event following the procedure. ${ }^{[21,22]}$ Postoperative complication rates vary by patient-related factors and procedurerelated factors.

\subsection{Surgical site infection}

Surgical site infection (SSI) is a potentially catastrophic complication of lumbar fusion. SSI can cause pseudarthrosis (i.e., failed fusion), neurologic injury, paralysis, sepsis, and death. ${ }^{[23]}$ SSI is also associated with prolonged length of stay, admission to intensive care, reoperation, and hospital readmission. ${ }^{[24-26]}$ In an effort to decrease the incidence of SSI, several interventions have been recommended. These include preoperative screening for nasal methicillin-resistant Staphylococcus aureus and decolonization using topical antibiotics and preadmission showering with chlorhexidine. ${ }^{[27,28]}$ Prophylactic antibiotic administration is also standard practice. Nevertheless, the incidence of SSI following spine surgery ranges from $0.7 \%$ to $14 \% .{ }^{[23,29]}$ Patients with diabetes mellitus are at particular risk of SSI due to several diabetes-related factors. Microvascular abnormalities impede oxygen and nutrient transport to peripheral tissues and cause tissue ischemia and delayed wound healing. High blood glucose levels im- pair immune system function. Diabetic peripheral neuropathy interferes with the normal inflammatory response. ${ }^{[26]}$ In addition to diabetes, advanced age (i.e., greater than 60 years), alcohol abuse, excessive blood loss, neurological injury, obesity, previous surgery, previous surgical infection, posterior approach fusion and APF, prolonged surgical time, and smoking have all been shown to increase patient risk of SSI. ${ }^{[29]}$

\subsection{Venous thromboembolism}

Venous thromboembolism (VTE) is another potentially devastating complication of lumbar fusion. VTE can present as deep vein thrombosis (DVT) or pulmonary embolism (PE), both of which are associated with increased morbidity, mortality, and prolonged length of stay. ${ }^{[30]}$ To help prevent the pooling of blood in a patient's legs during and after surgery, mechanical compression via pneumatic compression device or compression stockings is applied to the lower extremities prior to surgery and remains in place until the patient is fully ambulatory. ${ }^{[31]}$ Chemoprophylaxis may also be warranted for patients at high-risk of VTE, such as patients undergoing APF and patients with multiple trauma, malignancy, or hypercoagulable disorders. However, chemoprophylaxis must be carefully considered because it is associated with bleeding complications, including epidural hematoma and wound complications. ${ }^{[31,32]}$ For this reason, the Food and Drug Administration has mandated "black box" warnings on some anticoagulants for patients undergoing spinal procedures. ${ }^{[33]}$ Patients with medical comorbidities that increase their risk of VTE must be closely monitored. Older patients and patients with pulmonary circulation disorders, anemia, and fluid/electrolyte disorders are at increased risk of DVT and PE; patients with obesity and coagulopathy are at increased risk of DVT. ${ }^{[30]}$ Patients taking anticoagulants prior to fusion for non-spine related disorders, such as a heart valve replacement, must also be closely monitored and require "bridge" therapy to manage their anticoagulant regimen before and after lumbar fusion.

\subsection{Pseudarthrosis}

Pseudarthrosis refers to a failed fusion. Unlike other adverse events that present during the immediate or subacute recovery period, pseudarthrosis may not be evident for months or years following fusion surgery. Pseudarthrosis is also distinct from other complications in that patients with a failed fusion may remain asymptomatic. ${ }^{[34]}$ Metabolic abnormalities, infection, excess motion at the fusion site, and vitamin D deficiency all increase a patient's risk for pseudarthrosis. ${ }^{[34,35]}$ Cigarette smoking also increases a patient's risk for pseudarthrosis. Nicotine and carbon monoxide, among 
other toxins, impede the formation of new bone by interfering with oxygen transport, neovascularization, and osteoblast differentiation. ${ }^{[36]}$ Thus, all patients are strongly urged to cease smoking prior to fusion. Smoking cessation as little as 4 weeks prior to surgery has been shown to benefit wound healing and may favorably influence bone healing. [37]

\section{NuRSING CARE}

Nurses individualize patient care and education to each patient's unique spinal pathology, comorbidities, surgical risk factors, psychosocial context, and environment. Working in partnership with patients and the interprofessional team, nurses focus on optimizing patients' physical and mental health and mobilizing their social support systems.

Ideally, surgical planning commences at least several weeks prior to the scheduled procedure. During the diagnostic workup, nurses educate patients about the indications and implications of imaging and neurophysiological testing. Since patient safety is a priority, it is imperative to identify patients who may be pregnant to avoid imaging modalities that emit ionizing radiation. Patients with embedded or implanted devices must be screened prior to MR to accurately identify the device and determine MR compatibility. This process typically necessitates a review of written documentation from the device manufacturer, such as the results of formal testing of the implant before implantation or product labeling of the implant. ${ }^{[38]}$ Patients scheduled for MR should be encouraged to leave metallic personal belongings and devices (i.e., watches, jewelry, cell phones, metallic drug delivery patches) at home because they cannot be brought into the examination area owing to the strong MR magnet. Patients should also be aware that MR examination requires that they lie motionless inside a cylinder-shaped tube for upwards of 1 hour. If a patient anticipates difficulty tolerating this requirement due to severe anxiety or claustrophobia, a one-time dose of an anxiolytic or intravenous sedation may be appropriate. Prior to contrasted studies, the radiologist reviews the medical history and blood urea nitrogen and creatinine levels (assessed within 48 hours of the examination) to evaluate the risk of contrast-induced nephropathy. Following contrasted studies, patients are encouraged to increase oral fluid intake to help clear the contrast material. Patients should also be instructed to seek emergent medical attention if they develop hives, skin rash, itching, nausea, or headache following a contrasted study because they may be experiencing a hypersensitivity reaction to the contrast agent.

In advance of surgery, patients should be able to identify the reason for surgery, the intended surgical procedure, and the possible benefits of surgery. Patients must also be aware of the potential complications of surgery so that they approach

Published by Sciedu Press lumbar fusion with realistic expectations. They should be able to verbalize prescribed adjustments to their medication regimen (i.e., anticoagulant therapy, diabetes medications, herbal supplements) as well as food and fluid restrictions and skin and showering instructions. Patients should be aware of opportunities for autologous blood donation, smoking cessation assistance, and vitamin D supplementation. Clear expectations about postoperative care, including plans for pain management, diet, activity, physical therapy, pulmonary hygiene, criteria for discharge, anticipated length of stay, and the need to enlist a caregiver to assist during recovery, must be communicated. In addition, patients should be able to verbalize how and when to contact the health care team. ${ }^{[39]}$

Patients scheduled for elective lumbar fusion are admitted to the hospital on the morning of surgery. Once consents are signed and all preoperative testing is complete, nurses typically administer prophylactic antibiotics and apply mechanical compression devices to the patient's lower extremities. Once in the operating room, the anesthesiologist administers general endotracheal anesthesia to the patient. The surgical team then positions the patient and pads pressure points. A neurophysiologist may perform intraoperative neurophysiologic monitoring to identify neurological injury during the procedure. Vital signs, hemodynamic status, and intake and output are continuously monitored during the procedure. When the surgery is finished, the surgeon typically uses an antibiotic-impregnated solution to irrigate the wound. A surgical drain may be placed to facilitate wound drainage. The anesthesiologist then extubates the patient and the surgical team transports the patient to the post-anesthesia recovery unit for several hours. The patient is subsequently transferred to the surgical care unit for post-operative care until safe for discharge to home or a rehabilitation facility.

Nursing care during surgical recovery prioritizes neurovascular assessment, wound care, mobility, pulmonary hygiene, and pain management. Frequent neurological assessment is required to detect sensory and motor deficits. These deficits are usually transient, but are immediately reported to the surgical team so that the stability and integrity of the spinal column and spinal cord can be assessed. When there has been extensive manipulation of the nerves during surgery, steroid medication may be indicated to decrease inflammation and irritation of the nerve roots. However, prolonged postoperative steroid use should be avoided because it increases the risk of infection. ${ }^{[3]}$ Repeated vascular assessment is necessary to identify swelling, redness, increased warmth, or tenderness in the lower extremities that warrant evaluation to rule out DVT. Chest pain, shortness of breath, tachycardia, and tachypnea warrant evaluation to rule out PE. ${ }^{[40]}$ 
Diligent wound care is required to promote healing and to identify signs and symptoms of SSI. Increased wound drainage, erythema, pain, an elevated C-reactive protein level, or an elevated body temperature are immediately reported to the surgical team for further evaluation. Prior to hospital discharge, patients must be able to describe wound care and identify the signs and symptoms of SSI. This is important because more than $90 \%$ of SSIs are identified following hospital discharge. ${ }^{[41]}$ When SSI is diagnosed, patients often require a long course of antimicrobial therapy and possibly a return to the operating room for wound irrigation and debridement. Vacuum-assisted wound closure may also be utilized to facilitate healing. ${ }^{[23]}$ Signs and symptoms of a cerebral spinal fluid (CSF) leak can also be detected during wound care. CSF leaks occur when the dura is inadvertently punctured during surgery. If not detected intraoperatively, a CSF leak may present as a draining wound, a fluid collection, or a postural headache. When a dural tear is suspected, the nurse immediately notifies the surgical team and instructs the patient to assume a flat-lying position. If symptoms do not resolve within 24 to 48 hours, surgical exploration and placement of a sub-arachnoid drain may be indicated. ${ }^{[39]}$

Early postoperative ambulation is important to prevent immobility-related complications, such as skin breakdown, DVT, and pneumonia. When getting a patient out of bed, nurses instruct the patient to move to the side of the bed and assume a sitting position by gradually lowering the legs while raising the torso. Careful execution of this movement avoids twisting at the waist. A gradual increase in activity is encouraged, but patients should be instructed to refrain from bending, heavy lifting, and twisting until the surgical wound has healed and the fusion has ossified. ${ }^{[42]}$ In some instances, a lumbar brace is indicated to reduce flexion and extension of the lumbar spine.

Meticulous pulmonary hygiene assists patients to clear mucous and secretions from the lungs and prevent atelectasis and pneumonia. Nurses encourage frequent (i.e., hourly) deep breathing, coughing, and incentive spirometry. Appropriate pain management also promotes recovery. Current guidelines recommend multimodal analgesia that integrates a variety of analgesic medications and techniques (i.e., epidural with local anesthetic with or without opioid or intrathecal opioid) with non-pharmacologic interventions (i.e., cognitive modalities, relaxation, music) to manage postoperative pain. ${ }^{[43]}$ Nurses should use a validated pain assessment tool, such as the Numeric Rating Scale or the Wong-Baker FACES pain rating scale to measure pain intensity (see Figure $3){ }^{[44]}$ Whenever a patient is receiving opioid pain medication, nurses monitor the patient's level of consciousness and respiratory status to evaluate the efficacy of treatment and to identify adverse effects. ${ }^{[43]}$ In anticipation of discharge, nurses ensure that the patient is able to describe the pain management plan, including strategies to manage opioid-related adverse effects (i.e., constipation). Patients are instructed to gradually taper their opioid use. Many patients use opioids for months following lumbar fusion. ${ }^{[45,46]}$ However, prolonged opioid use should be discouraged because it may increase the risk of an opioid use disorder or drug poisoning death. $^{[47-49]}$

\section{Wong-Baker FACES ${ }^{\circledast}$ Pain Rating Scale}

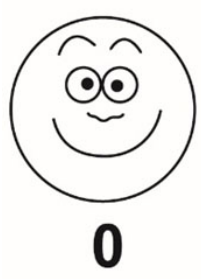

No

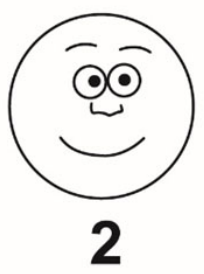

Hurts Little Bit

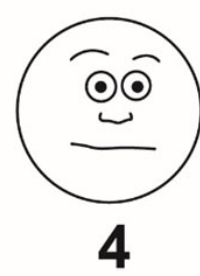

Hurts
ittle More

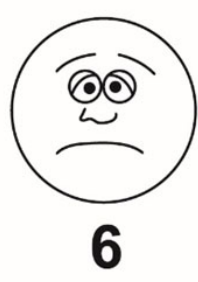

Hurts
Even More

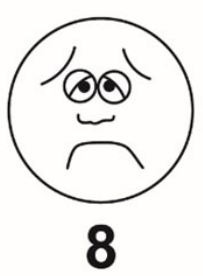

Hurts
Whole Lot

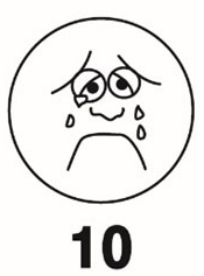

Hurts Worst

()1983 Wong-Baker FACES Foundation. www.WongBakerFACES.org

Used with permission. Originally published in Whaley \& Wong's Nursing Care of Infants and Children. (CEIsevier Inc.

Figure 3. Wong-Baker FACES $₫$ pain rating scale ${ }^{[44]}$

Following hospital discharge, nurses continue to play an important role in patient recovery. Nurses frequently manage telephone inquiries from patients and family members. Ac- cordingly, nurses must recognize the patient concerns that warrant immediate evaluation to rule out a surgical complication. Nurses may also be involved with referrals to home 
health nursing, physical therapy, and occupational therapy as well as outpatient rehabilitative services. During the first few weeks following lumbar fusion, therapy is limited to static stabilization exercises, such as pelvic tilts and bridging. These exercises allow patients to move their arms and legs without rocking or arching the lower trunk. As rehabilitation progresses, therapy incorporates dynamic exercises. These exercises may incorporate exercise balls and resistance bands to stretch and strengthen the muscles of the back and abdomen.

Finally, nurses must recognize the extent to which psychological and social factors affect patient recovery. Depressive symptoms, pain catastrophizing (i.e., negative thoughts about pain), and preoperative opioid dependence have all been linked to negative postsurgical outcomes. Patients with preoperative depressive symptoms have reported greater dissatisfaction and less improvement in disability following fusion than patients without depressive symptoms. ${ }^{[50,51]} \mathrm{Pa}-$ tients with high levels of preoperative pain catastrophizing have reported more postoperative pain and functional disability than patients who do not catastrophize. ${ }^{[52]}$ Among patients with work-related injuries, preoperative depression and preoperative opioid dependence have been associated with higher postoperative medical costs, lower return to work rates, lower work retention rates, and longer postoperative pain medication use compared to other patients. ${ }^{[3,54]} \mathrm{Al}-$ though additional research is needed to develop and evaluate

\section{REFERENCES}

[1] Hickey JV. The clinical practice of neurological and neurosurgical nursing. Philadelphia (PA): Wolters Kluwer; 2014. 816 p.

[2] Seicean A, Alan N, Seicean S, et al. Surgeon specialty and outcomes after elective spine surgery. Spine. 2014 Sep; 39(19): 1605-13. https://doi.org/10.1097/BRS.0000000000000489

[3] Skinner HB, McMahon PJ. Current diagnosis and treatment in orthopedics. Version 5. New York: McGraw-Hill; c2014 [cited 2017 Mar 23]. Available from: http://accessmedicine.mhmedical.com

[4] Chou R, Baisden J, Carragee EJ, et al. Surgery for low back pain: a review of the evidence for an American Pain Society Clinical Practice Guideline. Spine. 2009 May; 34(10): 1094-109. https: //doi.org/10.1097/BRS.0b013e3181a105fc

[5] Zaina F, Tomkins-Lane C, Carragee E, et al. Surgical versus nonsurgical treatment for lumbar spinal stenosis. Cochrane Database Syst Rev [Internet]. 2016 Jan 29 [cited 2017 Mar 23]; CD010264. https://doi.org/10.1002/14651858.CD010264.pub2

[6] Chen MM, Pope, TL, Ott DJ. Basic radiology [Internet]. 2nd ed. New York: McGraw-Hill; 2011 [cited 2017 Mar 23]. Available from: http://accessmedicine.mhmedical.com/

[7] International Society for the Advancement of Spine Surgery. Policy statement on lumbar spinal fusion surgery [Internet]. Aurora (IL): International Society for the Advancement of Spine Surgery; c2011 the efficacy of specific strategies to improve perioperative outcomes, conducting a holistic evaluation of every patient should enable nurses to recognize psychological and social barriers to recovery.

\section{SUMMARY}

Patients undergoing lumbar fusion have complex spinal pathology. Some patients have traumatic injuries, other patients have destabilizing spinal tumors or infection, and many patients have degenerative conditions. In addition, patients often present for lumbar fusion with comorbid medical conditions that increase the risk of a surgical adverse event. Thus, expert nursing care is an essential component of an interprofessional approach to patient management. Nurses with a strong foundation in lumbar spinal anatomy and pathology can interpret the diagnostic evaluation and identify surgical indications. Nurses who screen their patients for surgical risk factors can implement preventive measures. Nurses who are familiar with the various operative approaches to lumbar fusion can focus their nursing assessments and promptly recognize surgical complications. Finally, nurses who utilize the biopsychosocial model in their practice can recognize those patients for whom mental health or social work referral may be necessary to promote recovery and prevent long-term disability.

\section{CONFLiCTS OF INTEREST Disclosure}

The author reports no conflicts of interest to disclose. [cited 2017 Mar 23]. Available from: http://www.isass.org/pd f/2011-07-15_policy_statement_lumbar_surgery.pdf

[8] North American Spine Society. Lumbar fusion: defining appropriate coverage positions[Internet]. Burr Ridge (IL): North American Spine Society; c2016 [cited 2017 Mar 29]. Available from: https://www.spine.org/PolicyPractice/CoverageR ecommendations/.aspx

[9] Rajaee SS, Bae HW, Kanim LE, et al. Spinal fusion in the United States: analysis of trends from 1998 to 2008. Spine. 2012 Jan; 37(1): 67-76. https ://doi.org/10.1097/BRS.0b013e31820cccfb

[10] Phillips FM, Slosar PJ, Youssef JA, et al. Lumbar spine fusion for chronic low back pain due to degenerative disc disease: a systematic review. Spine. 2013 Apr; 38(7): E409-22. https ://doi.org/10 .1097/BRS.0b013e3182877f 11

[11] Anderson JT, Haas AR, Percy R, et al. Single-level lumbar fusion for degenerative disc disease is associated with worse outcomes compared with fusion for spondylolisthesis in a workers' compensation setting. Spine. 2015 Mar; 40(5): 323-31. https: //doi.org/10.1097/BRS.0000000000000734

[12] Brunicardi FC, Andersen DK, Billiar TR, et al. Schwartz's Principles of Surgery [Internet]. 10th ed. New York: McGraw-Hill, c2014 [cited 2017 Mar 23]. Available from: http://accessmedicine.mhmed ical.com/ 
[13] Talia AJ, Wong ML, Lau HC, et al. Comparison of the different surgical approaches for lumbar interbody fusion. J Clin Neurosci. 2015 Feb; 22(2): 243-51. https ://doi.org/10.1016/j.jocn. 2014. 08.008

[14] Goz V, Weinreb JH, Schwab F, et al. Comparison of complications, costs, and length of stay of three different lumbar interbody fusion techniques: an analysis of the Nationwide Inpatient Sample database. Spine J. 2014 Sep; 14(9): 2019-27. https : //doi.org/10.1016/ j.spinee.2013.11.050

[15] Winder MJ, Gambhir S. Comparison of ALIF vs. XLIF for L4/5 interbody fusion: pros, cons, and literature review. J Spine Surg. 2016 Mar; 2(1): 2-8. https://doi.org/10.21037/jss.2015.12.01

[16] Rao PJ, Loganathan A, Yeung V, et al. Outcomes of anterior lumbar interbody fusion surgery based on indication: a prospective study. Neurosurgery. 2015 Jan; 76(1): 7-23; discussion 23-4. https://doi.org/10.1227/NEU.0000000000000561

[17] Street JT, Andrew Glennie R, Dea N, et al. A comparison of the Wiltse versus midline approaches in degenerative conditions of the lumbar spine. J Neurosurg Spine. 2016 Sep; 25(3): 332-8. https://doi.org/10.3171/2016.2.SPINE151018

[18] Adogwa O, Parker SL, Bydon A, et al. Comparative effectiveness of minimally invasive versus open transforminal lumbar interbody fusion: 2- year assessment of narcotic use, return to work, disability, and quality of life. J Spinal Disorders Tech. 2011 Dec; 24(8): 479-84 https://doi.org/10.1097/BSD.0b013e3182055cac

[19] Goldstein CL, Macwan K, Sundararajan K, et al. Perioperative outcomes and adverse events of minimally invasive versus open posterior lumbar fusion: meta-analysis and systematic review. J Neurosurg Spine. 2016 Mar; 24(3): 416-27. https ://doi .org/10.3171/20 15.2.SPINE14973

[20] Peng CW, Yue WM, Poh SY, et al. Clinical and radiological outcomes of minimally invasive versus open transforaminal lumbar interbody fusion. Spine. 2009 Jun; 34(13): 1385-9. https : //doi .org/10.1 097/BRS. 0b013e3181a4e3be

[21] Cheng I, Briseno MR, Arrigo RT, et al. Outcomes of two different techniques using the lateral approach for lumbar interbody arthrodesis. Global Spine J. 2015 Aug; 5(4): 308-14. https ://doi .org/ $10.1055 / \mathrm{s}-0035-1546816$

[22] Joseph JR, Smith BW, La Marca F, et al. Comparison of complication rates of minimally invasive transforaminal lumbar interbody fusion and lateral lumbar interbody fusion: a systematic review of the literature. Neurosurg Focus. 2015 Oct; 39(4): 11. https://doi.org/10.3171/2015.7.FOCUS15278

[23] Radcliff KE, Neusner AD, Millhouse PW, et al. What is new in the diagnosis and prevention of spine surgical site infections. Spine $\mathrm{J}$. 2015 Feb; 15(2): 336-47. https : //doi.org/10.1016/j.spinee .2014 .09 .022

[24] Chen S, Anderson MV, Cheng WK, et al. Diabetes associated with increased surgical site infections in spinal arthrodesis. Clin Orthop Relat Res. 2009 Jul; 467(7): 1670-3. https ://doi .org/10.100 7/s11999-009-0740-y

[25] Koutsoumbelis S, Hughes AP, Girardi FP, et al. Risk factors for postoperative infection following posterior lumbar instrumented arthrodesis. J Bone Joint Surg Am. 2011 Sep; 93(17): 1627-33. https://doi.org/10.2106/JBJS.J.00039

[26] Zhang Y, Zheng QJ, Wang S, et al. Diabetes mellitus is associated with increased risk of surgical site infections: a meta-analysis of prospective cohort studies. Am J Infect Control. 2015 Aug; 43(8): 810-5. https://doi.org/10.1016/j.ajic.2015.04.003

[27] Thakkar V, Ghobrial GM, Maulucci CM, et al. Nasal MRSA colonization: impact on surgical site infection following spine surgery.
Clin Neurol Neurosurg. 2014 Oct; 125: 94-7. https : //doi .org/ 10.1016/j.clineuro.2014.07.018

[28] Edmiston CE, Lee CJ, Krepel CJ, et al. Evidence for a standardized preadmission showering regimen to achieve maximal antiseptic skin surface concentrations of chlorhexidine gluconate, 4\%, in surgical patients. JAMA Surg. 2015 Nov; 150(11): 1027-33. https ://doi .org/10.1001/jamasurg . 2015.2210

[29] North American Spine Society. Evidence-based clinical guidelines for multidisciplinary spine care: antibiotic prophylaxis in spine surgery [Internet]. Burr Ridge (IL): North American Spine Society; c2007 [revised 2013; cited 2017 Mar 23]. Available from: https ://www.spine.org/Portals/0/Documents/Research ClinicalCare/Guidelines/AntibioticProphylaxis.pdf

[30] Fineberg SJ, Oglesby M, Patel AA, et al. The incidence and mortality of thromboembolic events in lumbar spine surgery. Spine. 2013 Jun; 38(13): 1154-9. https://doi.org/10.1097/BRS.0b013e3182 $86 \mathrm{~b} 7 \mathrm{c} 0$

[31] North American Spine Society. Evidence-based clinical guidelines for multidisciplinary spine care: antithrombotic therapies in spine surgery [Internet]. Burr Ridge (IL): North American Spine Society; c2009 [cited 2017 Mar 28]. Available from: https: //www .spine.org/Portals/0/Documents/ResearchClinic alCare/Guidelines/AntithromboticTherapies.pdf

[32] Schroeder GD, Kurd MF, Kepler CK, et al. Postoperative epidural hematomas in the lumbar spine. J Spinal Disord Tech. 2015 Nov; 28(9): 313-318. https://doi.org/10.1097/BSD.0000000000 000329

[33] Sanofi-Aventis. Lovenox (enoxaparin sodium injection): full prescribing information [Internet]. Bridgewater (NJ): c2009 [cited 2017 jul 27]. Available from: https://www .accessdata.fda.gov/d rugsatfda_docs/label/2009/020164s0851bl.pdf

[34] Raizman NM, O’Brien JR, Poehling-Monaghan KL, et al. Pseudarthrosis of the spine. J Am Acad Orthop Surg. 2009 Aug; 17(8): 494-503. PMid:19652031 https://doi.org/10.5435/001246 35-200908000-00003

[35] Kerezoudis P, Rinaldo L, Drazin D, et al. Association between vitamin D deficiency and outcomes following spinal fusion surgery: a systematic review. World Neurosurg. 2015 Nov; 95: 71-76. https : //doi.org/10.1016/j.wneu.2016.07.074

[36] Bydon M, De la Garza-Ramos R, Abt NB, et al. The impact of smoking on complication and pseudoarthrosis rates following single and two-level posterolateral fusion of the lumbar spine. Spine. $2014 \mathrm{Oct}$; 39(21): 1765-1770. https://doi.org/10.1097/BRS.00000000 00000527

[37] Truntzer J, Vopat B, Feldstein M, et al. Smoking cessation and bone healing: optimal cessation timing. Eur J Orthop Surg Traumatol. 2015 Feb; 25(2): 211-5. https ://doi.org/10.1007/s00590-0 $14-1488-y$

[38] Kanal E, Barkovich AJ, Bell C, et al. ACR guidance document on MR safe practices. J Magn Reson Imaging. 2013 Mar; 37(3): 501-530. https://doi.org/10.1002/jmri.24011

[39] American Association of Neuroscience Nurses. Thoracolumbar spine surgery: a guide to preoperative and postoperative patient care [Internet]. Chicago (IL): American Association of Neuroscience Nurses; c2014 [cited 2016 July 1]. Available from: http: //www . aann . org /pubs/content/guidelines.html

[40] Evans NS, Ratchford EV. Vascular disease patient information page: venous thromboembolism (deep vein thrombosis and pulmonary embolism). Vasc Med. 2014 Apr; 19(2): 148-150. https : //doi.org/10.1177/1358863X14529007

[41] Haleem A, Chiang HY, Vodela R, et al. Risk factors for surgical site infections following adult spine operations. Infect Control Hosp 
Epidemiol. 2016 Dec; 37(12): 1458-67. https ://doi.org/10.1 017/ice.2016.193

[42] Greenwood J, McGregor A, Jones F, et al. Evaluating rehabilitation following lumbar fusion surgery (REFS): study protocol for a randomised controlled trial. Trials. 2015 Jun; 16(1): 8. https : //doi.org/10.1186/s13063-015-0751-9

[43] Chou R, Gordon DB, de Leon-Casasola OA, et al. Management of postoperative pain: a clinical practice guideline from the American Pain Society, the American Society of Regional Anesthesia and Pain Medicine, and the American Society of Anesthesiologists' Committee on Regional Anesthesia, Executive Committee, and Administrative Council. J Pain. 2016 Feb; 17(2): 131-57. https://doi.org/10.1016/j.jpain.2015.12.008

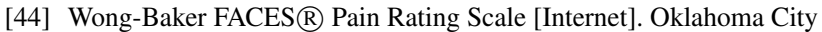
(OK): Wong-Baker FACES Foundation; c2016 [cited 2017 Feb 22]. Available from: http://www. WongBakerFACES . org

[45] Nguyen TH, Randolph DC, Talmage J, et al. Long-term outcomes of lumbar fusion among workers' compensation subjects: a historical cohort study. Spine. 2011 Feb; 36(4): 320-31. https ://doi .org/ 10.1097/BRS. 0b013e3181ccc220

[46] Rouben D, Casnellie M, Ferguson M. Long-term durability of minimal invasive posterior transforaminal lumbar interbody fusion: a clinical and radiographic follow-up. J Spinal Disord Tech. 2011 Jul; 24(5): 288-96. https://doi.org/10.1097/BSD.0b013e3181f9a60a

[47] Freynhagen R, Geisslinger G, Schug SA. Opioids for chronic noncancer pain. BMJ. 2013 May; 346. https ://doi.org/10.1136/ bmj.f 2937
[48] Labianca R, Sarzi-Puttini P, Zuccaro SM, et al. Adverse effects associated with non-opioid and opioid treatment in patients with chronic pain. Clin Drug Investig. 2012 Feb; 32 Suppl 1: 53-63. https://doi.org/10.2165/11630080-000000000-00000

[49] Paulozzi LJ, Zhang K, Jones CM, et al. Risk of adverse health outcomes with increasing duration and regularity of opioid therapy. J Am Board Fam Med. 2014 May-Jun; 27(3): 329-38. https : //doi.org/10.3122/jabfm.2014.03.130290

[50] Adogwa O, Carr K, Fatemi P, et al. Psychosocial factors and surgical outcomes: are elderly depressed patients less satisfied with surgery? Spine. 2014 Sep; 39(19): 1614-9. https ://doi .org/10.1097/BR S. 0000000000000474

[51] Adogwa O, Parker SL, Shau DN, et al. Preoperative Zung Depression Scale predicts outcome after revision lumbar surgery for adjacent segment disease, recurrent stenosis, and pseudarthrosis. Spine J. 2012 Mar; 12(3): 179-85. https://doi.org/10.1016/j.spinee.2 011.08 .014

[52] Abbott AD, Tyni-Lenne R, Hedlund R. Leg pain and psychological variables predict outcome 2-3 years after lumbar fusion surgery. Eur Spine J. 2011 Oct; 20(10): 1626-34. https://doi .org/10.1007/ s00586-011-1709-6

[53] Anderson JT, Haas AR, Percy R, et al. Clinical depression is a strong predictor of poor lumbar fusion outcomes among workers' compensation subjects. Spine. 2015 May; 40(10): 748-56. https://doi.org/10.1097/BRS.0000000000000863

[54] Mayer TG, Gatchel RJ, Brede E, et al. Lumbar surgery in workrelated chronic low back pain: can a continuum of care enhance outcomes? Spine J. 2014; 14(2): 263-73. https ://doi.org/10.1 $016 / j \cdot$ spinee. 2013.10 .041 